\title{
Concomitant Regulation by a Lacl-Type Transcriptional Repressor XyIR on Genes Involved in Xylan and Xylose Metabolism and the Type III Secretion System in Rice Pathogen Xanthomonas oryzae pv. oryzae
}

\author{
Yumi Ikawa, ${ }^{1}$ Sayaka Ohnishi, ${ }^{1}$ Akiko Shoji, ${ }^{1}$ Ayako Furutani, ${ }^{2}$ and Seiji Tsuge ${ }^{1,+}$ \\ ${ }^{1}$ Laboratory of Plant Pathology, Graduate School of Agriculture, Kyoto Prefectural University, Kyoto 606-8522, Japan; and \\ ${ }^{2}$ Gene Research Center, Ibaraki University, Inashiki 300-0393, Japan
}

Accepted 20 January 2018.

\begin{abstract}
The hypersensitive response and pathogenicity ( $h r p)$ genes of Xanthomonas oryzae pv. oryzae, the causal agent of bacterial leaf blight of rice, encode components of the type III secretion system and are essential for virulence. Expression of hrp genes is regulated by two key hrp regulators, HrpG and HrpX; HrpG regulates $h r p X$ and $h r p A$, and $\mathrm{HrpX}$ regulates the other $h r p$ genes on $h r p B$-hrpF operons. We previously reported the sugar-dependent quantitative regulation of HrpX; the regulator highly accumulates in the presence of xylose, followed by high hrp gene expression. Here, we found that, in a mutant lacking the LacI-type transcriptional regulator XylR, HrpX accumulation and $h r p$ gene expression were high even in the medium without xylose, reaching the similar levels present in the wild type incubated in the xylose-containing medium. XylR also negatively regulated one of two xylose isomerase genes (xylA2 but not $x y l A 1)$ by binding to the motif sequence in the upstream region of the gene. Xylose isomerase is an essential enzyme in xylose metabolism and interconverts between xylose and xylulose. Our results suggest that, in the presence of xylose, inactivation of XylR leads to greater xylan and xylose utilization and, simultaneously, to higher accumulation of $\mathrm{HrpX}$, followed by higher hrp gene expression in the bacterium.
\end{abstract}

Xanthomonas oryzae pv. oryzae is the causal agent of a rice disease, bacterial leaf blight (Niño-Liu et al. 2006; Swings et al. 1990). The bacterium enters rice leaves from hydathodes or wounds at leaf edges and grows in xylem vessels (Ou 1985). Generally, phytopathogenic bacteria have evolved a highly efficient protein secretion system, called the type III secretion system (T3SS), which is the important device to directly introduce type III effector proteins (T3Es) into plant cells (Büttner and Bonas 2010). The T3SS and some of the T3Es are encoded on clustered hypersensitive response and pathogenicity ( $h r p$ ) genes, which are essential for bacterial pathogenicity on host plants and are required to induce the hypersensitive

${ }^{\dagger}$ Corresponding author: Seiji Tsuge; E-mail: s_tsuge@kpu.ac.jp

Funding: This work was supported by Japan Society for the Promotion of Science KAKENHI grant number 16K07621.

*The $\boldsymbol{e}$-Xtra logo stands for "electronic extra" and indicates that eight supplementary figures and two supplementary tables are published online.

(c) 2018 The American Phytopathological Society response (HR) on nonhost plants (Alfano and Collmer 1997; Büttner and Bonas 2002).

The expression of hrp genes in plant-pathogenic bacteria is highly regulated and is only induced in plants or certain nutrient-poor synthetic media, so-called hrp-inducing media (Arlat et al. 1991; Huang et al. 1991; Schulte and Bonas 1992; Wengelnik et al. 1996b). In Xanthomonas bacteria, the hrp gene cluster comprises six operons ( $h r p A$ to $h r p F$ ), and the expression of the genes is regulated by two key hrp regulators HrpG and HrpX. Protein HrpG is predicted to be a two-component response regulator, belonging to the OmpR family, and activates the expression of $h r p X$ and $h r p A$ (Li et al. 2014; Wengelnik and Bonas 1996; Wengelnik et al. 1996a). Then, HrpX, which is an AraC-type transcriptional regulator, activates the expression of other $h r p$ operons ( $h r p B$ to $h r p F$ ) (Wengelnik and Bonas 1996).

Recently, HpaS, a putative cognate sensor kinase for HrpG, was identified in the brassica pathogen $X$. campestris pv. campestris, and a mutant lacking HpaS had greatly reduced phosphorylation of HrpG and expression of $h r p X$ and $h r p G(\mathrm{Li}$ et al. 2014). In addition, a specific point mutation in $\mathrm{HrpG}$, which makes the protein constitutively active without phosphorylation, results in hrp gene expression even in the non-hrp inducing condition in $X$. campestris pv. vesicatoria (Wengelnik et al. 1999) and also in X. oryzae pv. oryzae (S. Tsuge unpublished data). Therefore, although the homolog of HpaS is not encoded on the genome of $X$. oryzae pv. oryzae and the sensor kinase for HrpG remains unknown, phosphorylation of HrpG is thought to be an important step for hrp gene expression in the bacterium. In addition, transcriptional activation of $h r p G$ is also important. So far, factors such as the histone-like nucleoidstructuring proteins XrvA and XrvB, the GntR family protein Trh, and two-component systems PhoP/PhoQ and RaxH/RaxR are known to be involved in hrpG expression (Burdman et al. 2004; Feng et al. 2009; Kametani-Ikawa et al. 2011; Lee et al. 2008; Tsuge et al. 2006).

We recently reported that the sugar source is an important factor for inducing the expression of $h r p$ genes in $X$. oryzae pv. oryzae (Ikawa and Tsuge 2016). In addition, although hrp regulators $\mathrm{HrpG}$ and $\mathrm{HrpX}$ are expressed independently of any sugar sources in the medium, the accumulation of HrpX and the expression of HrpX-regulated hrp genes are high only in the presence of xylose. Thus, posttranslational, quantitative regulation of a second key hrp regulator, HrpX, probably in a proteolytic manner, is another essential step for hrp gene expression, and xylose plays an important role in suppressing this 
negative regulation. However, the factors involved in the regulatory cascade are unknown.

The LacI-type transcriptional regulator XylR, which consists of a ligand-binding domain and a DNA-binding domain, functions as a repressor of xylan and xylose utilization genes in X. campestris pv. campestris (Déjean et al. 2013). The regulator is well conserved, not only in xanthomonads but, also, in a wide range of bacterial genera. In the aquatic bacterium Caulobacter crescentus, XylR binds to a specific DNA motif in the promoter region of target genes in the absence of $x y-$ lose but dissociates from the operator when it is bound to xylose, leading to gene expression to induce xylose metabolism (Hottes et al. 2004; Meisenzahl et al. 1997; Stephens et al. 2007).

In the present report, we show that XylR in $X$. oryzae pv. oryzae negatively regulates not only the expression of a xylose metabolism-related gene but, also, the accumulation of the hrp regulator HrpX. In the absence of XylR, the accumulation of HrpX is independent of xylose, resulting in higher hrp gene expression even in the xylose-free condition.

\section{RESULTS}

Generation of a mutant with high hrp gene expression that is independent of xylose.

To identify the gene involved in xylose-dependent $h r p$ gene expression in $X$. oryzae pv. oryzae, we conducted transposon mutagenesis using the EZ::TN<KAN2> system (Tsuge et al.
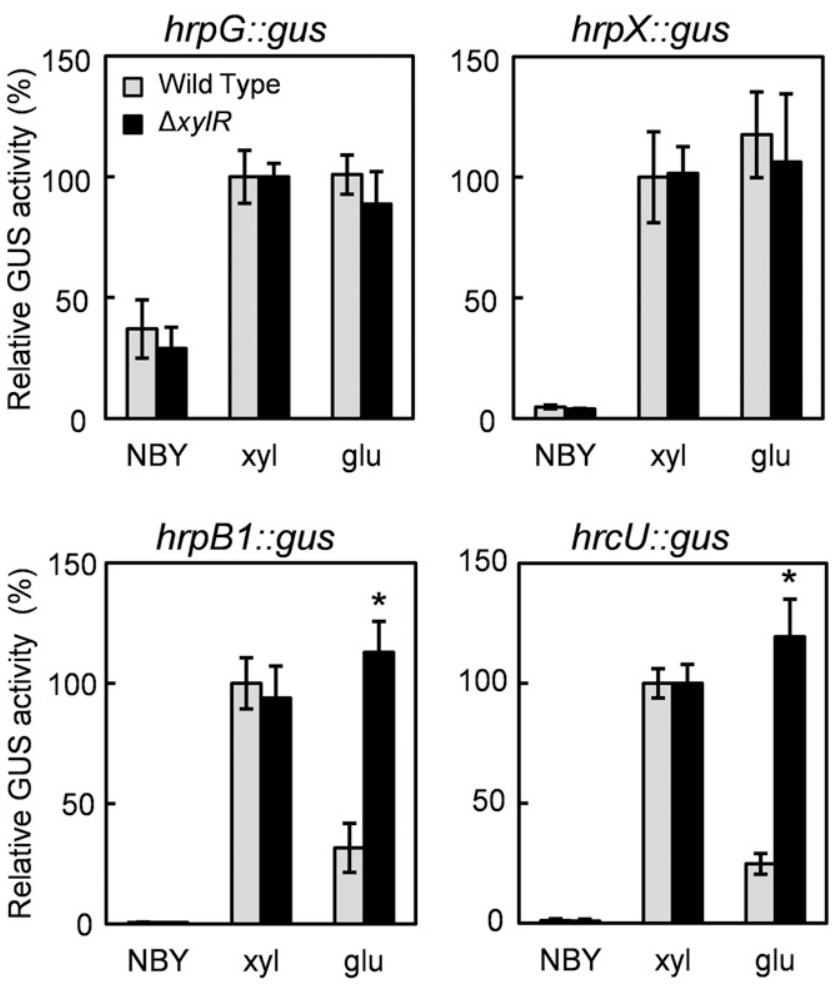

Fig. 1. Mean ( \pm standard deviation) relative $\beta$-glucuronidase (GUS) activity of Xanthomons oryzae pv. oryzae wild type (gray bars) and the XylR mutant $(\Delta x y l R)$ (black bars) transformed with the plasmid harboring hrpG::gus, hrpX::gus, hrpB1::gus, or hrcU::gus after incubation in XOM2 (with xylose [xyl]), XOM2-based glucose medium (glu), or nutrient-rich complex medium NBY. Values are relative to that of the wild-type background incubated in XOM2, from four replicates of each combination of medium and transformant. Similar results were obtained in two independent experiments. Asterisks indicate that values of the mutant significantly differed from that of the wild type as determined by a $t$ test (one asterisk [ $\left.{ }^{*}\right]$ indicates $P<0.01)$ using the Gen-Infoweb server.
2004) and parental strain 74Hpa1::Lux (Rashid et al. 2016), which harbors the lux operon derived from Vibrio fischeri (O'Kane and Prasher 1992), controlled by the promoter of hpal and, thus, bioluminesces in the $h r p$-inducing condition. Approximately 1,500 kanamycin-resistant clones were then incubated on two media, i.e., the hrp-inducing medium XOM2, which contains xylose, and a modified XOM2 that contains glucose instead of xylose and induces less hrp expression than $\mathrm{XOM} 2$ does. A clone was found to produce a similar intensity of bioluminescence on the two media (Supplementary Fig. S1). Sequence analysis of the transposon-inserted region, followed by a homology search of the genome database of $X$. oryzae $\mathrm{pv}$. oryzae MAFF311018 (Ochiai et al. 2005), revealed that a transposon was inserted into +201 of XOO_4161 (+1 is A of the putative initiation codon) (Supplementary Fig. S2). According to the domain search program of the National Center for Biotechnology Information web server, the gene is predicted to encode a protein with features typical of a LacI-type transcriptional regulator, a helix-turn-helix DNA-binding domain and a ligand-binding domain. The homolog of XOO_4161 in $X$. campestris pv. campestris (XCC_4101, 94\% identity) was annotated as XylR and was shown to be a repressor for a large proportion of genes that encode the xylan and xylose utilization system (Déjean et al. 2013). Following the usage in the paper by Déjean et al. (2013), we also named XOO_4161 as $x y l R$.

\section{$\mathrm{XylR}$ is involved in negative regulation}

on the expression of HrpX-regulated hrp genes.

We generated an in-frame deletion mutant, $\Delta x y l R$, in which the sequence comprising positions +97 to $+1,048$ of $x y l R$ (952 bp of the 1,068-bp gene) was deleted. Then the expression of HrpX-regulated $h r p$ genes $h r p B 1$ and $h r c U$ ( $h r p B 1$ and $h r c U$ are the first genes of the $h r p B$ and $h r p C$ operons, respectively) in $\Delta x y l R$ was examined after incubation in XOM2 (with xylose) or the XOM2-based glucose medium (glucose instead of xylose was added). Plasmid harboring hrpB $1:: g u s$ or $h r c U:: g u s$ preceded by the promoter region of each operon was introduced into $X$. oryzae pv. oryzae wild-type MAFF311018 and the XylR mutant, and $\beta$-glucuronidase (GUS) activity in each transformant was measured after a 19-h incubation. As shown in Figure 1, GUS activity in any of the wild-type derivatives with any of the hrp/hrc::gus fusion genes was highly reduced in the glucose medium compared with those in the xylose medium, as we already reported (Ikawa and Tsuge 2016), while the activity in each of the mutant backgrounds in the glucose medium was significantly higher than in the wild type and reached a level similar to those in the xylose medium. We also examined the expression of $h r p G$ and $h r p X$ using a GUS reporter assay; HrpG regulates $h r p X$ expression, and HrpX regulates the expression of other $h r p$ genes, including $h r p B 1$ and $h r c U$. We previously reported that the expression of $h r p G$ and $h r p X$ is sugarindependent, unlike $h r p X$-regulated $h r p$ genes (Ikawa and Tsuge 2016). Here, for both regulator genes, GUS activity was similar among the wild type and the XylR mutant in the xylose medium XOM2 and in the XOM2-based glucose medium (Fig. 1).

To confirm the increased expression of HrpX-regulated hrp gene in the XylR mutant in the glucose medium, we examined the accumulation of hrp gene transcripts in the mutant and wild type by real-time quantitative reverse transcription-polymerase chain reaction (qRT-PCR) after a 6-h incubation in the XOM2based glucose medium. As shown in Figure 2, the accumulation of HrpX-regulated $h r c U$ transcripts was much higher in the mutant lacking XylR than in the wild type, while $h r p G$ and $h r p X$ transcript levels were similar between two strains. Introduction of the complementary plasmid pHMXylR into the mutant decreased $h r c U$ transcript level to that in the wild type. 
The results were completely consistent with the data from the GUS reporter assay.

\section{Accumulation of HrpX is high in XylR mutant even when xylose is absent.}

In previous reports, we revealed that hrp gene expression is induced by incubation in the nutrient-poor and xylosecontaining medium and that the induction is canceled when the sugar source is replaced with other sugars, such as glucose. In the medium with other sugars, the accumulation of HrpX is reduced, probably by proteolysis (Ikawa and Tsuge 2016; Tsuge et al. 2002). In $\Delta x y l R, h r p$ gene expression was high even in the medium containing glucose, reaching a level similar to that in the xylose-containing medium (Figs. 1 and 2). This finding led us to examine the accumulation of HrpX in the XylR mutant after incubation in XOM2 with either xylose or glucose. Proteins isolated from bacteria incubated in each medium for $19 \mathrm{~h}$ were separated with sodium dodecyl sulfatepolyacrylamide gel electrophoresis (SDS-PAGE), then, were analyzed by Western blot using an anti-HrpX polyclonal antibody. In $\Delta x y l R$, the level of HrpX was high even after incubation in the xylose-free glucose medium, similar to the level after incubation in the xylose-containing medium, although the quantity of protein in the wild type was lower in the glucose medium than in the xylose medium (Fig. 3). The introduction of the complementary plasmid to the mutant resulted in less HrpX accumulation in the glucose medium. These results suggest that the higher expression of hrp genes in the XylR mutant after culturing in the glucose medium is caused by the increased accumulation of HrpX and that XylR is involved in the sugardependent quantitative regulation of a key hrp regulator, HrpX.

\section{Expression of XylR}

in xylose- and glucose-containing media.

To examine whether the expression of XylR differs between the xylose- and the glucose-containing media, we constructed a plasmid harboring a gus gene preceded by the putative promoter region of $x y l R$ and introduced it into the wild-type strain. After a 19-h incubation of the strain in XOM2 or the XOM2based glucose medium, GUS activity was measured. Similar

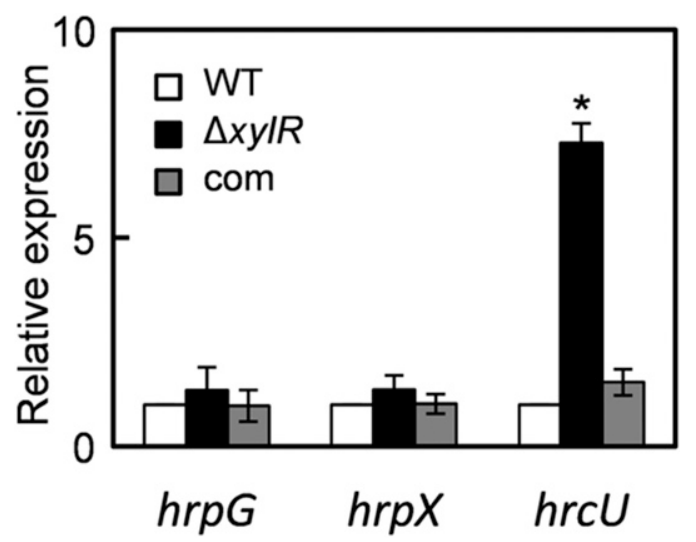

Fig. 2. Real-time quantitative reverse transcription-polymerase chain reaction analysis of accumulation of $h r p G, h r p X$, and $h r c U$ transcripts in Xanthomonas oryzae pv. oryzae strains incubated in the XOM2-based glucose medium. Mean ( \pm standard deviation) mRNA levels of each gene in the mutant and complementary strains relative to that in the wild type were calculated from five (for $h r p G$ and $h r p X$ ) or three (for $h r c U$ ) measurements. $\mathrm{WT}=$ wild type with the empty vector, $\Delta x y l R=$ XylR mutant with the empty vector, and com $=$ XylR mutant with complementary plasmid pHMXylR. An asterisk indicates that values of the mutant significantly differed from that of the wild type as determined by a $t$ test (one asterisk [ ${ }^{*}$ ] indicates $P<0.01)$.
GUS activity was observed between the two sugar conditions, suggesting that the involvement of XylR in the sugar-dependent quantitative regulation of $\operatorname{HrpX}$ is due to the sugar-dependent function of XylR, not to its expression (Fig. 4).

\section{XylR also regulates the expression}

of $x y l A 2$ encoding xylose isomerase.

In $X$. campestris pv. campestris, XylR represses the expression of genes related to xylan and xylose utilization, including one of two putative xylose isomerase genes, $x y l A 2$, that is located next to $x y l R$ and predicted to encode an enzyme that catalyzes the interconversion of xylose and xylulose (Déjean et al. 2013). In $X$. oryzae pv. oryzae, $x y l A 2$ is also located next to $x y l R$. Therefore, we examined whether XylR of $X$. oryzae pv. oryzae is also involved in the negative regulation of xylA 2 expression by measuring GUS activity in the wild type and in the XylR mutant transformed with the plasmid harboring a gus gene preceded by the putative promoter region of $x y l A 2$ after their incubation in the XOM2-based glucose medium with or without xylose. Similar GUS activity was observed in the mutant between two sugar conditions, while the activity in the wild type incubated without xylose was significantly lower than that with xylose (Fig. 5A). The higher expression of $x y l A 2$ in $\Delta x y l R$ was also tested by real-time qRT-PCR analysis. Here, we used primer set XylA2_RT_S and XylA1/XylA2_RT_AS, which amplified a fragment corresponding to -67 to +236 of $x y l A 2$, not an internal fragment of the gene, because $x y l A 1$ (XOO_2761) and $x y l A 2$ are completely identical with each other. As shown in Figure 5B, higher accumulation of the $x y l A 2$ transcript in the mutant than the wild type was confirmed and the level of the transcript in the complementary strain was similar to that in the wild type.

We also examined the expression of $x y l A l$ in the presence or absence of XylR. Genes xylA1 (XOO_2761) and the preceding $X O O \_2762$ are very closely located (47-bp gapped). RT-PCR analysis, using total RNA prepared from the wild-type strain incubated in XOM2 as a template and primer set XylA1_RT_S and XylA1/XylA2_RT_AS, which hybridize internal sequences of $X O O \_2762$ and $x y l A 1$, respectively, successfully amplified the fragment containing the $3^{\prime}$ region of $X O O \_2762$ and the $5^{\prime}$ region of $x y l A 1$, suggesting that the two genes are in an operon (Supplementary Fig. S3). Therefore, a real-time qRTPCR analysis using the same primer set was conducted for the wild type, the XylR mutant, and the complementary strain after incubation in the XOM2-based glucose medium. Unlike for $x y l A 2$, the $x y l A 1$ transcript level in the XylR mutant did not differ significantly from that in the wild type (Fig. 5B). These

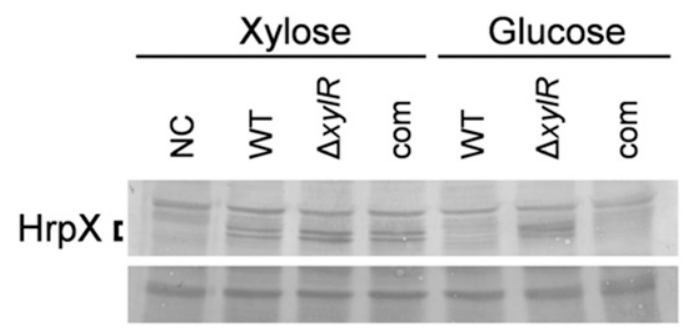

Fig. 3. Western blot analysis of HrpX accumulation in Xanthomonas oryzae pv. oryzae wild type (WT), XylR mutant $(\Delta x y l R)$, and the complementary strain (com) incubated in xylose-containing XOM2 and XOM2-based glucose medium. Negative control $(\mathrm{NC})=$ MAFF $\Delta h r p X($ HrpX-deficient mutant) incubated in XOM2. After electrophoresis and blotting, the upper part of the membrane (including HrpX) was used for Western blot analysis using an anti-HrpX antibody. Here, two HrpX-specific signals are detected as previously reported (Ikawa and Tsuge 2016). The lower part (not including HrpX) was stained with Coomassie brilliant blue. Similar results were obtained in two independent experiments. 
results suggest that $X$. oryzae pv. oryzae XylR negatively regulates only one of two xylose isomerase genes, xylA2, located next to $x y l R$, but is not involved in the regulation of the other, $x y l A 1$.

We examined the possibility that XylR-regulated XylA2 is involved in hrp gene expression. The deletion mutant of xylA2 transformed with pHMhrcU::gus showed similar GUS activity to the wild type with the same plasmid. The result suggests that $\mathrm{XylR}$ is involved in the regulation of $h r p$ gene expression independently of the regulation of $x y l A 2$ and XylA2-mediated xylose metabolism (Supplementary Fig. S4).

\section{XylR directly binds to the promoter of $x y l A 2$.}

We investigated the mechanism by which XylR regulates $x y l A 2$ in $X$. oryzae pv. oryzae. In $C$. crescentus, XylR binds to a specific DNA motif upstream of the xylose metabolism-related gene operon to repress transcription in the absence of xylose, but it dissociates from the operator to allow gene transcription in the presence of xylose (Hottes et al. 2004; Meisenzahl et al. 1997; Stephens et al. 2007). A sequence similar to this XylR-binding motif in $C$. crescentus was previously found in the promoter

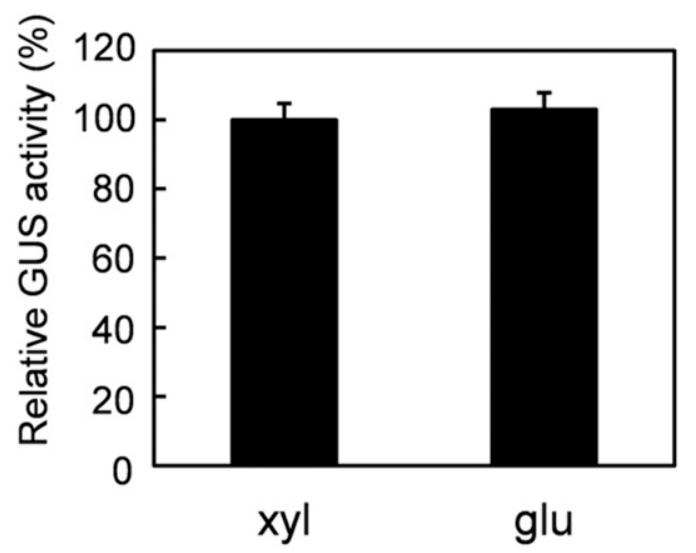

Fig. 4. Mean ( \pm standard deviation) relative $\beta$-glucuronidase (GUS) activity of Xanthomonas oryzae pv. oryzae strains transformed with the plasmid harboring $x y l R::$ gus and incubated in XOM2 (xyl) or XOM2-based glucose medium (glu). Values are relative to that in XOM2 with xylose from four replicates. Similar results were obtained in two independent experiments. region of xylA2 in $X$. campestris pv. campestris (Déjean et al. 2013) and here in $X$. oryzae pv. oryzae (Supplementary Fig. S5). We then examined the binding activity of $X$. oryzae pv. oryzae XylR to the $x y l A 2$ promoter region and its importance in XylR-dependent regulation of $x y l A 2$ expression.

An Escherichia coli strain was transformed with pETXylRHis, which produces the full-length XylR protein tagged with six histidine residues at the $\mathrm{C}$ terminus (XylR-His), was incubated in Luria-Bertani (LB) broth (Sambrook et al. 1989), and protein production was induced by adding isopropyl- $\beta$-Dthiogalactopyranoside (IPTG), followed by purifying the protein using a nickel column (Supplementary Fig. S6). When the 413-bp DNA fragment from position -390 to +23 of $x y l A 2$, amplified by PCR with primer set XylA2_GUS_S and XylA2_5_AS, was incubated with XylR-His for $1 \mathrm{~h}$ at $25^{\circ} \mathrm{C}$ and then subjected to electrophoresis, the mobility of the DNA was specifically retarded compared with that of the DNA without the protein (Fig. 6). For the nonspecific control DNA, we used a fragment (approximately 1,000 bp long) containing the promoter region of a hybrid type two-component sensor/regulator gene stoS (Ikawa et al. 2014), which is expressed independently of XylR (data not shown). The control DNA was not retarded even with the XylR-His (Fig. 6). The results suggest that XylR specifically and directly binds to the $x y l A 2$ promoter region.

To further investigate the importance of XylR and its binding to the promoter region in $x y l A 2$ expression, we introduced base substitutions in the 14-bp motif sequence that was predicted to be the target of XylR. Fragments with base substitutions at three different positions (two inside the motif sequence and the other outside) were amplified and were used for the electrophoretic mobility shift assay. Although the mobility of the DNAs with no base substitution or with the base substitution outside the motif sequence shifted after the addition of XylRHis protein, that of the two fragments with base substitutions inside the motif did not shift, indicating the importance of the 14-bp motif for XylR binding (Fig. 7). Also, we constructed plasmids harboring a gus gene preceded by the $x y l A 2$ promoter region with or without base substitutions inside or outside the 14-bp motif (pHMXylA2::gus without base substitution, pHMXylA2M1::gus and pHMXylA2M2::gus with base substitutions inside the motif, and pHMXylA2M3::gus with base substitutions outside the motif) and introduced the respective
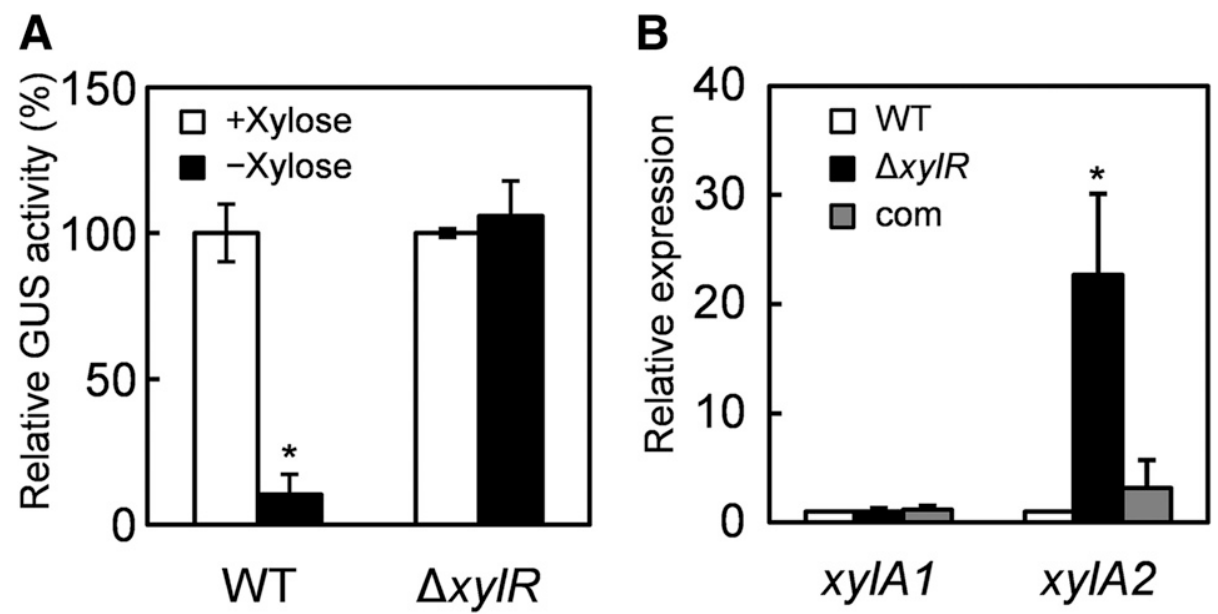

Fig. 5. A, Mean ( \pm standard deviation $[\mathrm{SD}]$ ) relative $\beta$-glucuronidase (GUS) activity of Xanthomonas oryzae pv. oryzae wild type (WT) and XylR mutant $(\Delta x y l R)$ transformed with the plasmid harboring $x y l A 2: \because g u s$ after incubation in the XOM2-based glucose medium with (+Xylose) (white bars) or without xylose (-Xylose) (black bars). Values are relative to that in the medium with xylose from four replicates. Similar results were obtained in two independent experiments. B, Real-time quantitative reverse transcription-polymerase chain reaction analysis of expression of $x y l A 1$ and $x y l A 2$ in $X$. oryzae pv. oryzae strains, wild type (WT), XylR mutant $(\Delta x y l R)$, and the complementary strain (com) incubated in XOM2-based glucose medium. Mean $( \pm$ SD) mRNA levels of each gene after incubation relative to that in the wild type were calculated by the comparative quantitation method (Warton et al. 2004) from triplicate measurements. Asterisks indicate a significant difference as determined by a $t$ test (one asterisk $[*]$ indicates $P<0.01$ ) using the Gen-Info web server. 
plasmids into the wild-type strain. After incubation in the XOM2-based glucose medium, GUS activity was significantly higher in strains with pHMXylA2M1::gus or pHMXylA2M2:: gus, but activity in the strain with pHMXylA2M3::gus remained low, similar to the level in the strain with pHMXylA2::gus (Fig. 8). GUS activity in the wild-type strain with pHMXylA2M1::gus and pHMXylA2M2::gus was much higher than in the XylR mutant with pHMXylA2::gus. We speculate that the base substitution might also prevent one or more other unknown negative regulators from binding to the $x y l A 2$ promoter. The results above indicate that $X$. oryzae $\mathrm{pv}$. oryzae XylR directly and negatively regulates the expression of $x y l A 2$ by binding the 14-bp motif sequence, which is likely to be well-conserved in a broad range of bacteria.

\section{Virulence of the $x y l R$ deletion mutant.}

Susceptible rice cultivar IR24 was inoculated with a bacterial suspension, and lesion lengths were measured 14 days later. As shown in Supplementary Figure S7, no significant difference in virulence was observed between the wild type and $\Delta x y l R$. In the infection process, xylose in rice leaves may inactivate the negative regulator XylR and, as a result, virulence of the mutant may be comparable to that of the wild type.

\section{DISCUSSION}

The expression of hrp genes, followed by the construction of the type III protein secretion system and secretion of effector proteins via that system have been revealed to be regulated by complex regulatory networks (Büttner and Bonas 2010; Piqué et al. 2015; Tsuge et al. 2014). In X. oryzae pv. oryzae, two key hrp regulators, HrpG and HrpG-regulated HrpX, play essential roles in hrp gene expression (Wengelnik and Bonas 1996; Wengelnik et al. 1996a). Moreover, several regulators that are involved in transcriptional regulation of $h r p G$ and an unknown sensor kinase that phosphorylates the response regulator HrpG are important members of the regulatory networks (Burdman et al. 2004; Feng et al. 2009; Kametani-Ikawa et al. 2011; Lee et al. 2008; Tsuge et al. 2006). Also, sugars (carbohydrates) and sugar metabolisms are likely to be closely associated with the regulation of $h r p$ gene expression. We recently reported that a LysR-type transcriptional regulator of galactose metabolismrelated genes, named GamR, also regulates the transcription of two key $h r p$ regulators, $h r p G$ and $h r p X$, by directly binding to an upstream region common to the genes, which are located next to each other in opposite directions (Rashid et al. 2016). Guo et al. (2012) reported that positive regulation of $h r p G$ and

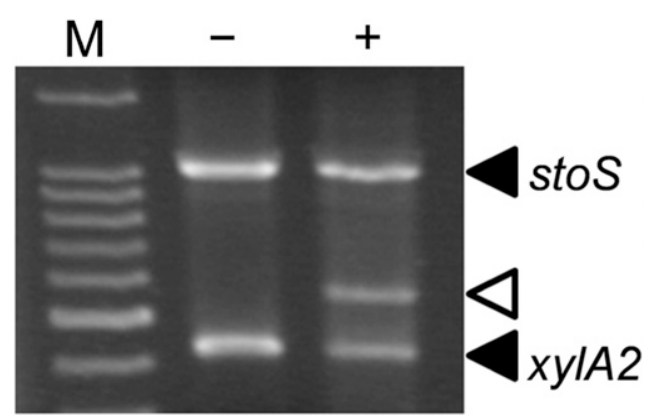

Fig. 6. DNA-binding assay of XylR-His protein. Mixture of DNA fragments containing the $x y l A 2$ promoter region or the stoS promoter region (used as a nonspecific control DNA) with $(+)$ or without $(-)$ XylR-His in $20-\mu 1$ reaction solution was separated by electrophoresis in $1.5 \%$ agarose gel and Tris-borate-EDTA buffer after a 1-h incubation at $25^{\circ} \mathrm{C}$. A white triangle indicates the shifted fragment of the $x y l A 2$ promoter region. M: 100-bp DNA ladder marker. $h r p X$ expression is associated with fructose-bisphophate aldolase, which catalyzes the reversible conversion of fructose1,6-bisphosphate into dihydroxyacetone phosphate and glyceraldehyde 3-phosphate in the glycolytic and gluconeogenic pathways in $X$. oryzae pv. oryzicola, which is genetically very close to X. oryzae pv. oryzae. Moreover, we recently found that the control of HrpX accumulation, probably through proteolysis, is an important regulatory step for hrp gene expression and that xylose can induce high accumulation of the regulator, although the detail mechanisms have been unclear (Ikawa and Tsuge 2016; Tsuge et al. 2002). In the present study, we showed that the LacI-type transcriptional repressor XylR, which is likely to regulate xylan and xylose utilization genes, is a key regulator of the xylose-dependent quantitative control of HrpX in $X$. oryzae pv. oryzae.

Xylan is the predominant hemicellulose in rice cell walls (Takeuchi et al. 1994) and is digested by endo-1,4- $\beta$-xylanase and xylan 1,4- $\beta$-xylosidase into its component xylose units. Xylose is converted to xylulose by a xylose isomerase and is then phosphorylated to xylulose-5-phosphate by xylulokinase, for entry into the pentose phosphate cycle. Acquisition and utilization of xylose is likely to be important for virulence of $X$. oryzae pv. oryzae. Xylanase $\mathrm{XynB}$ is involved in virulence (Rajeshwari et al. 2005) and a mutant with a transposon in the phosphoglucose isomerase gene, which cannot grow in the medium with xylose as a sole sugar source, is attenuated in virulence (Tsuge et al. 2004). Blanvillain et al. (2007) reported that $X$. campestris pv. campestris has a xylan CUT (carbohydrate utilization with TonBdependent outer membrane transporters [TBDTs]) system, which is involved in plant xylan and xylose scavenging and comprises enzymes that degrade xylan, inner membrane transporters, transcriptional regulators, and TBDTs. Also, in X. oryzae pv. oryzae, genes involved in the system are well-conserved. Xylose taken into the bacteria may be used as a nutrient, and, as our previous (Ikawa and Tsuge 2016) and present works indicate, it also functions as an inducer of hrp gene expression.

A
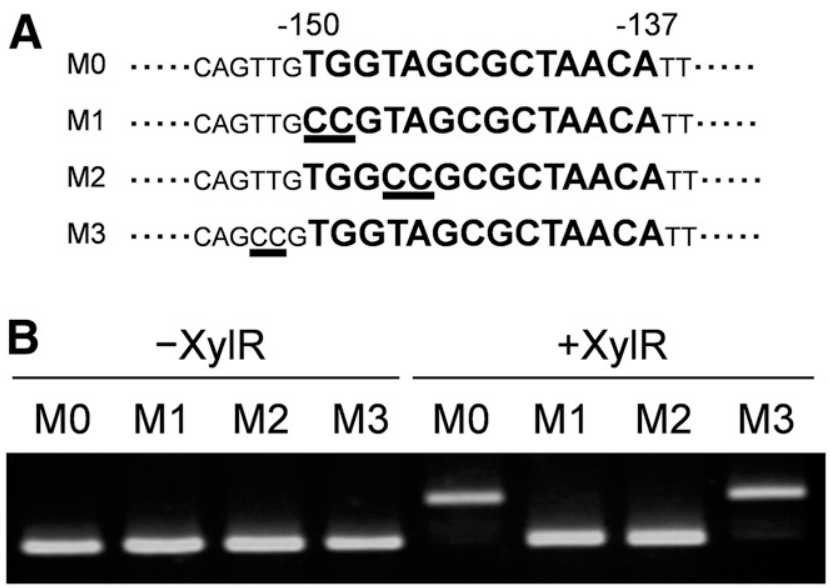

Fig. 7. DNA-binding assay of XylR-His protein using fragments containing the $x y l A 2$ promoter region with or without base substitutions. A, Nucleotide sequences with base substitutions. Bold letters represent the conserved 14bp palindromic motif that could be targeted by XylR, located at position -150 to -137 from the start codon of xylA2. Base substitutions inside (M1 and M2) or outside (M3) the motif are underlined. Fragment M0 contains no base substitution. B, Electrophoretic mobility shift assay using fragments M0 to M3 shown in A as templates. A mixture of each DNA fragment with or without XylR-His in $20-\mu$ l reaction solution was separated by electrophoresis in $1.5 \%$ agarose and Tris-borate-EDTA buffer after a 1-h incubation at $25^{\circ} \mathrm{C}$. For preventing nonspecific binding of XylR-His to template DNAs, the DNA fragment containing the stoS promoter region was also added to the reaction mixture but is not shown here. 
The regulator XylR is well-conserved, not only in Xanthomonas spp. but also in a wide range of bacterial genera, and it negatively regulates expression of xylan and xylose utilization genes by directly binding to the well-conserved 14-bp palindromic motif in the promoter region of the target genes or operons (Déjean et al. 2013; Gärtner et al. 1992; Hottes et al. 2004; Stephens et al. 2007). In X. campestris pv. campestris, the XylR-binding motif was found in the promoter region of four operons or genes putatively involved in xylan, xylose, or glucuronic acid utilization (Déjean et al. 2013). We confirmed that all four operons or genes in $X$. oryzae pv. oryzae were also preceded by the motif and that

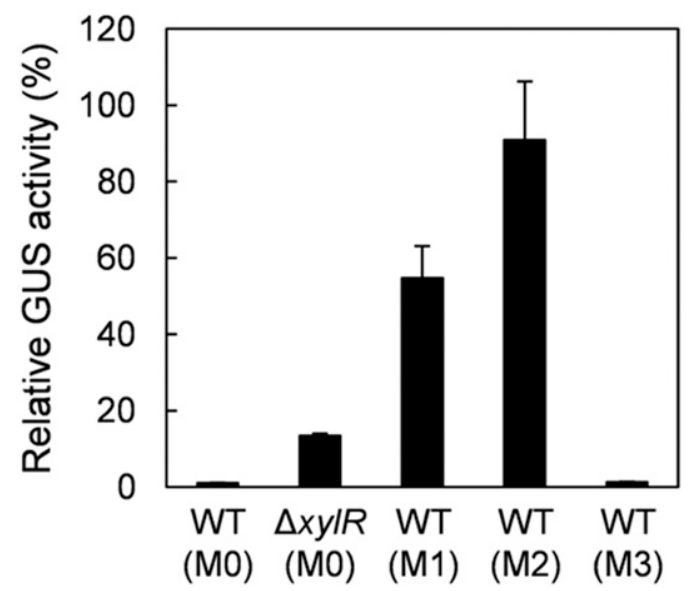

Fig. 8. $\beta$-glucuronidase (GUS) activity of Xanthomonas oryzae pv. oryzae strains with a plasmid harboring the gus gene preceded by the (non) basesubstituted promoter region of $x y l A 2$ after incubation in the XOM2-based glucose medium. WT $=$ wild type and $\Delta x y l R=$ XylR mutant. M0, M1, M2, and M3 in parentheses correspond to strains harboring the plasmid containing the (non) base-substituted sequence. A $t$ test using the Gen-Info web server revealed significant differences $(P<0.01)$ among all samples except for the combination between WT (M0) and WT (M3). at least one xylose isomerase gene, $x y l A 2$, was regulated dependently via XylR and the XylR-binding motif sequence (Figs. $5,6,7$, and 8). These facts suggest that XylR also functions as a regulator for xylan and xylose utilization in $X$. oryzae pv. oryzae.

In previous papers, xylose has been thought to serve as a ligand of XylR and that binding of xylose to XylR leads to dissociation of the regulator from the target DNA sequences in C. crescentus (Hottes et al. 2004; Meisenzahl et al. 1997; Stephens et al. 2007). Although our data showing that the addition of xylose leads to the expression of XylR-regulated xylA2 suggests the involvement of xylose in dissociation of XylR from the target motif also in X. oryzae pv. oryzae (Fig. 5), we could not prove it. In our experiment, XylR was not released from the motif sequence in the $x y l A 2$ promoter region by the addition of xylose, even at the concentration of $50 \mathrm{mM}$, similarly to the equivalent test with glucose as a negative control (Supplementary Fig. S8). This unexpected result may be due to the experimental conditions. Alternatively, a certain metabolite from xylose might function as the ligand of XylR. X. oryzae pv. oryzae possesses two identical xylose isomerase genes, xylAI and $x y l A 2$, and unlike that of $x y l A 2$, the expression of $x y l A 1$ is XylR-independent (Fig. 5B). Xylose metabolism could be started by XylA1 and a metabolite might dissociate XylR from the promoter region of $x y l A 2$, followed by the expression of the gene and more active xylose metabolism.

When the bacterium enters rice leaves from hydathodes or wounds at leaf edges and grows in xylem vessels, nutrient acquisition and suppression of host defense responses are important for survival and spread in host plants. Xylem vessels are nutrient-poor, but xylose or xylan is abundant in rice cell walls and could provide a sugar source easily acquired as free xylose or through the digestion of xylan by the bacterial xylan CUT system-dependent or -independent xylanase and xylosidase. Under such a condition, it is likely that xylose is a key factor for growth and virulence of $X$. oryzae pv. oryzae. We show the regulatory model proposed for XylR and genes involved in

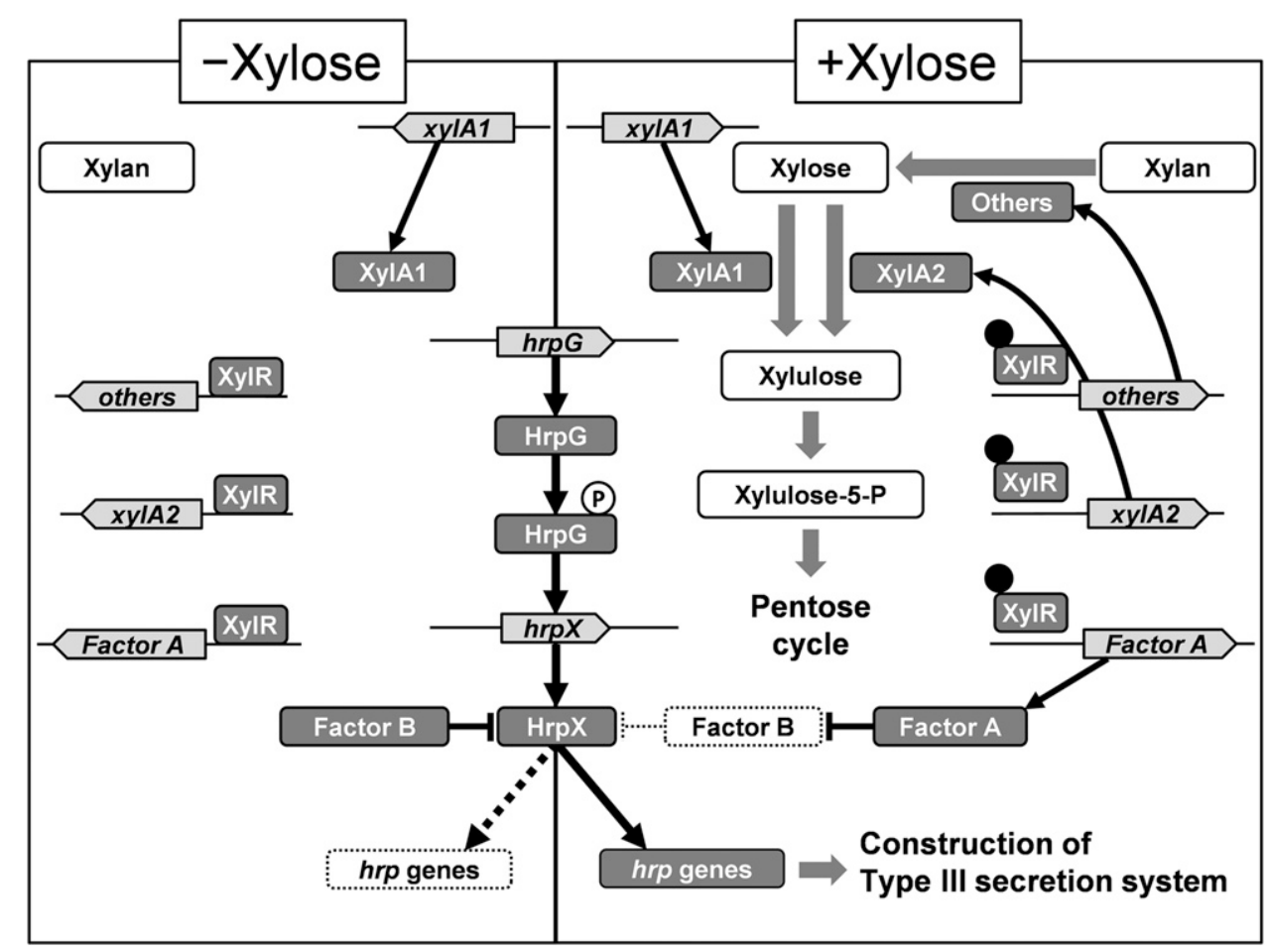

Fig. 9. Regulatory model proposed for XylR and genes involved in xylose metabolism and the construction of the type III secretion system. Black circles represent xylose or its derivative that binds to XylR to dissociate the protein from the target DNAs. 
xylose metabolism and the construction of the T3SS in Figure 9. In the presence of xylose, xylose is converted to xylulose by xylose isomerase XylA1, which is produced in a XylR-independent manner and is then used as a nutrient through the pentose cycle. And at the same time, xylose or its derivative binds to XylR to dissociate the protein from the target DNAs, resulting in i) activation of gene expression involved in xylan utilization (Déjean et al. 2013), ii) production of an 'extra' xylose isomerase XylA2 for more effective xylan and xylose utilization, enabling bacteria to grow more actively, and, at the same time, iii) production of unknown factor $\mathrm{A}$, which suppresses expression or activity of the other unknown factor B that is directly involved in lowering accumulation of HrpX. Inactivation of factor B leads to greater accumulation of HrpX and, then, to activation of hrp gene expression for construction of the T3SS and for secretion of T3Es to suppress host defense responses. In the absence of xylose, XylR remains on the promoter region of the target genes to repress their expression, leading to less or no expression of xylan utilizationrelated genes, $x y l A 2$ and the factor A gene. And because factor A is not functioning, factor B actively functions to reduce HrpX accumulation, thus lowering expression of hrp genes.

As major virulence factors of $X$. oryzae pv. oryzae, AvrXa7, PthXo1, PthXo2, and PthXo3 are reported, which are T3Es and belong to a transcriptional activator-like (TAL) effector family (Antony et al. 2010; Chen 2014; Chen et al. 2010; Chu et al. 2006; Yang et al. 2006; Zhou et al. 2015). Unlike other effectors that suppress host defense responses, they activate the expression of sugar transporter genes of rice. Therefore, after $h r p$ genes are xylose-dependently expressed and T3Es are secreted into rice cells, the sugar transported from rice cells by the action of TAL effectors may be mainly utilized by the bacteria as nutrients, leading to explosive bacterial growth in rice leaves.

As shown above and in Figure 9, regulation of HrpX accumulation by XylR must be mediated by at least two unknown factors, A and $\mathrm{B}$. The expression of factor $\mathrm{A}$ is directly and negatively regulated by XylR, and the expression or the function of factor B, which is probably a protease, is negatively regulated by factor $\mathrm{A}$, and then, factor B is directly involved in less accumulation of HrpX (Fig. 9). We found that at least four genes or operons are preceded by the XylR-binding motif-like sequence, which includes xylan and xylose utilization gene operons identified in $X$. campestris pv. campestris (Déjean et al. 2013) (data not shown). Among them, XylA2 was not involved in regulation of $h r p$ gene expression. One of the others might be factor A, which is regulated by XylR and regulates factor B. Identification of genes or factors that mediate XylR-dependent regulation of HrpX accumulation is now underway.

\section{MATERIALS AND METHODS}

\section{Bacterial strains, plasmids, and growth conditions.}

Bacterial strains and plasmids used in this study are listed in Supplementary Table S1. E. coli strains were cultured at $37^{\circ} \mathrm{C}$ in LB medium (Sambrook et al. 1989). X. oryzae pv. oryzae strains were usually grown at $28^{\circ} \mathrm{C}$, using nutrient broth-yeast extract (NBY) medium (Vidaver 1967). The nutrient-poor synthetic medium XOM2 (Tsuge et al. 2002) containing $0.18 \%$ xylose as a sole sugar source was used for $h r p$ induction. A modified XOM2 medium that contains $0.18 \%$ glucose in place of xylose (XOM2-based glucose medium) was also used. All media were supplemented with the following antibiotics, when required: ampicillin, $50 \mu \mathrm{g} \mathrm{ml}^{-1}$ for $E$. coli; kanamycin, $25 \mu \mathrm{g} \mathrm{ml}^{-1}$ for $X$. oryzae pv. oryzae and $50 \mu \mathrm{g} \mathrm{ml}^{-1}$ for $E$. coli; and spectinomycin, $25 \mu \mathrm{g} \mathrm{ml}^{-1}$ for $X$. oryzae pv. oryzae and E. coli.

\section{Transposon mutagenesis.}

An EZ::TN $<\mathrm{KAN} 2>$ transposome, a mixture of the transposon EZ::TN<KAN2> and EZ::TN transposase (Epicentre, Madison,
WI, U.S.A.), was directly introduced by electroporation into $X$. oryzae pv. oryzae $74 \mathrm{Hpa} 1:$ :Lux, a strain that produces bioluminescence under the control of the HrpX-regulated hpal promoter (Rashid et al. 2016), as described previously (Tsuge et al. 2004). Kanamycin-resistant clones were selected by plating, and the bioluminescence of each clone was recorded as described below and was compared with that of the parental strain 74Hpa1::Lux.

\section{Bioluminescence measurement.}

Bioluminescent strains of $X$. oryzae pv. oryzae were preincubated on NBY agar overnight and were then transferred to the hrp-inducing medium XOM2 (Tsuge et al. 2002) and the XOM2based glucose medium using toothpicks, followed by incubation at $28^{\circ} \mathrm{C}$ for $48 \mathrm{~h}$. The bioluminescence signal from each bacterial colony was collected for $1 \mathrm{~min}$, using a video-intensified microscope camera and the Argus-100 system (Hamamatsu Photonics, Hamamatsu, Japan). Alternatively, bacteria preincubated on NBY agar were suspended in distilled water (DW), then, $5 \mu \mathrm{l}$ of each bacterial suspension, adjusted to a concentration of absorbance at $600 \mathrm{~nm}\left(A_{600}\right)=1.0$, were dropped onto XOM2 and the XOM2-based glucose medium, then were further incubated and bioluminescence was observed as described above.

\section{Sequence analysis.}

A dye terminator cycle sequencing reaction was conducted using a BigDye Terminator version 3.1 cycle sequencing kit (Applied Biosystems, Foster, CA, U.S.A.) according to the manufacturer's instructions, followed by electrophoresis and analysis with an autosequencer (model 373A; Applied Biosystems).

\section{PCR.}

PCR was conducted using DNA polymerase Blend Taq (Toyobo, Osaka, Japan) or KOD plus (Toyobo) according to the manufacturer's instructions. The primers used in this study are listed in Supplementary Table S2.

\section{Generation of mutant and complemented strains.}

For constructing the plasmid used to generate the $x y l R$ deletion mutant, the 1,011-bp upstream region of $x y l R$, including a 96-bp coding region, and the 892-bp downstream regions of the gene, including a 20-bp coding region, were amplified by PCR using $X$. oryzae pv. oryzae MAFF311018 genomic DNA as the template. Then, the fragments, the kanamycin-resistance gene from the EZ::TN $<\mathrm{KAN} 2>$ transposon and the levansucrase gene ( $s a c$ ) from pK18mobsacB (Schäfer et al. 1994) were serially cloned into pUC19 to generate pUC $\Delta X y l R:: \mathrm{Km}^{\mathrm{r}} / \mathrm{sac}$. The plasmid was introduced into MAFF311018 by electroporation, and the $x y l R$ mutant, named $\Delta x y l R$, which lacks an internal 952-bp fragment in the 1,068-bp coding region of the gene, was generated through two consecutive homologous recombination events.

For constructing the complementation plasmid of $x y l R$, a fragment containing the gene with the 369-bp upstream and the 57-bp downstream regions was amplified and was cloned into a broad host-range vector pHM1 (Innes et al. 1988). The obtained plasmid was introduced to $\Delta x y l R$ by electroporation.

The $x y l A 2$ deletion mutant was also generated similarly to $\Delta x y l R$. The 1,038-bp upstream region of xylA2, including a 23bp coding region, and the 1,061-bp downstream regions of the gene, including a 22-bp coding region, were amplified. Then the fragments, the kanamycin-resistance gene and the sac gene were serially cloned into pBluescript II $\mathrm{KS}+$ to generate $\mathrm{pBS} \Delta \mathrm{XylA} 2:: \mathrm{Km}^{\mathrm{r}} / \mathrm{sac}$. The plasmid was then used to generate the $x y l A 2$ mutant named $\Delta x y l A 2$, which lacks an internal 1,293bp fragment in the 1,338-bp coding region of the gene, through two consecutive homologous recombination events. 


\section{GUS reporter assay.}

Bacterial strains with a plasmid harboring a gus reporter gene preincubated on NBY agar overnight were washed and were suspended with DW at $A_{600}=1.0$ and $15 \mu \mathrm{l}$ was then added to $1.5 \mathrm{ml}$ of NBY medium or $150 \mu \mathrm{l}$ was added to $1.5 \mathrm{ml}$ of XOM2 or the XOM2-based glucose medium. After incubation at $28^{\circ} \mathrm{C}$ for $19 \mathrm{~h}$ with shaking (150 rpm), GUS activity was measured as previously described (Jefferson et al. 1987; Kametani-Ikawa et al. 2011). One unit of GUS activity was defined as nanomoles of $p$-nitrophenol released per hour. Simultaneously, the concentration of bacterial cellular proteins per assay was also examined. The bacterial cells in $1 \mathrm{ml}$ of culture were pelleted and were resuspended with $100 \mu \mathrm{l}$ of B-PER bacterial protein extraction reagent (Thermo Fisher Scientific, Waltham, MA, U.S. A.) to extract bacterial proteins. Protein concentrations were measured using the Protein Assay kit (Bio-Rad, Hercules, CA, U.S.A.), with bovine serum albumin as a reference. GUS activity of each sample was calculated as units per microgram of bacterial cellular proteins.

\section{Real-time qRT-PCR.}

Total RNA was extracted from bacteria using an RNeasy mini kit (Qiagen, Hilden, Germany). The quality of the RNA sample was confirmed by agarose gel electrophoresis, then, cDNA was synthesized from $0.2 \mu \mathrm{g}$ of each RNA sample, using the reverse transcriptase ReverTra Ace (Toyobo). Real-time qRT-PCR was performed using the Rotor-Gene Q system (Qiagen) and KOD SYBR qPCR mix (Toyobo), according to the manufacturers' instructions. The expression level of 16S rRNA was used for an internal standard, and the comparative quantitation method (Warton et al. 2004) was used to calculate the relative mRNA levels of the mutant and complementary strains with respect to the corresponding transcripts in the wild type. Significant differences in mRNA levels were determined by $t$ test (one asterisk [*] indicates $P<0.01$ ), using the Osaka University Gen-Info web server.

\section{Western blot analysis.}

Bacterial strains preincubated on NBY agar were suspended in DW at $A_{600}=1.5$, and then, $300 \mu$ of the suspension were added to $3 \mathrm{ml}$ of XOM2 or the XOM2-based glucose medium and were cultured at $28^{\circ} \mathrm{C}$ with shaking $(150 \mathrm{rpm})$ for $19 \mathrm{~h}$. Bacterial cells were pelleted and resuspended in $150 \mu \mathrm{l}$ of B-PER bacterial protein extraction reagent (Thermo Fisher Scientific) to extract bacterial proteins. Protein concentrations were measured using the Protein Assay kit (Bio-Rad) with bovine serum albumin as a reference, and samples of $10 \mu \mathrm{g}$ of protein were separated by SDS-PAGE. After electrophoresis, proteins were transferred to a membrane (Immobilon P; Merck, Darmstadt, Germany) and the upper part (including HrpX) was used for Western blot analysis, using a rabbit polyclonal antibody against the full-length of HrpX as a primary antibody and an alkaline phosphatase-conjugated antirabbit immunoglobulin $\mathrm{G}$ as a secondary antibody (Bio-Rad). The lower part of the membrane (not including HrpX) was stained with Coomassie brilliant blue. As a negative control, the protein sample prepared from HrpX-deficient mutant MAFF $\Delta h r p X$ (Tsuge et al. 2001) incubated in XOM2 was used.

\section{Preparation of XylR tagged with histidine residues.}

A NcoI-HindIII fragment containing the coding region of $x y l R$, which was amplified by PCR with primer set XylR_His_S and XylR_His_AS, was cloned in the expression vector pET28b (Merck) to generate pETXylR-His. E. coli BL21 (DE3) (Merck) transformed with the plasmid was preincubated at $37^{\circ} \mathrm{C}$ on $\mathrm{LB}$ plate overnight, then was suspended with sterilized DW to adjust the concentration of $A_{600}=1.0$. One milliliter of the suspension was transferred into $50 \mathrm{ml}$ of fresh LB broth. After a 3-h incubation at $37^{\circ} \mathrm{C}$, IPTG was added at a final concentration of $0.1 \mathrm{mM}$, and the bacteria were further incubated at $30^{\circ} \mathrm{C}$ for $1 \mathrm{~h}$. Bacterial cells were collected and were suspended in $20 \mathrm{ml}$ of equilibration buffer (50 mM sodium phosphate buffer [pH 7.4] with $300 \mathrm{mM} \mathrm{NaCl}$ and $20 \mathrm{mM}$ imidazole) and were sonicated. After the addition of $0.05 \%$ (final concentration) Tween 20, the bacterial suspension was centrifuged to separate the soluble and insoluble fractions, and XylR tagged with six histidine residues at the $\mathrm{C}$ terminus (XylR-His) in the soluble fraction was purified, using a His60 Ni gravity column purification kit (Clontech Laboratories, Mountain View, CA, U.S.A.) according to the manufacturer's instruction. The protein solution was bufferexchanged in $10 \mathrm{mM}$ Tris- $\mathrm{HCl}(\mathrm{pH} \mathrm{7.5)}$ containing $150 \mathrm{mM}$ $\mathrm{KCl}, 0.1 \mathrm{mM}$ dithiothreitol, $0.1 \mathrm{mM}$ EDTA, and $20 \%$ glycerol using a PD10 column (GE Healthcare, Buckinghamshire, U.K.) and stored at $-20^{\circ} \mathrm{C}$.

\section{Electrophoretic mobility shift assay.}

Approximately $200 \mathrm{ng}$ of template DNA and $120 \mathrm{ng}$ of XylRHis protein were mixed in $20 \mu \mathrm{l}$ of reaction solution containing a buffer (10 mM Tris- $\mathrm{HCl}$ [pH 7.5], $150 \mathrm{mM} \mathrm{KCl,} 2.5 \mathrm{mM}$ $\mathrm{MgCl}_{2}, 0.1 \mathrm{mM}$ dithiothreitol, and $0.1 \mathrm{mM}$ EDTA at final concentrations). When dissociation of the XylR-ligand complex from the target DNA sequence was examined, 0, 10, 20, and $50 \mathrm{mM}$ glucose or xylose was added. After incubation at $25^{\circ} \mathrm{C}$ for $1 \mathrm{~h}$, mixtures were separated by electrophoresis in $1.5 \%$ agarose gel in $0.5 \times$ Tris-borate-EDTA buffer. The DNA bands were visualized by staining with ethidium bromide.

\section{Virulence test.}

Bacterial strains grown on NBY-agar medium were washed twice and were adjusted to a concentration of $\mathrm{A}_{600}=0.03$ with DW. Susceptible rice cultivar IR24 was inoculated with the bacterial suspension by leaf clipping (Kauffman et al. 1973), and the lesion lengths were measured 14 days after inoculation.

\section{LITERATURE CITED}

Alfano, J. R., and Collmer, A. 1997. The type III (Hrp) secretion pathway of plant pathogenic bacteria: Trafficking harpins, Avr proteins, and death. J. Bacteriol. 179:5655-5662.

Antony, G., Zhou, J., Huang, S., Li, T., Liu, B., White, F., and Yang, B. 2010. Rice $x a 13$ recessive resistance to bacterial blight is defeated by induction of the disease susceptibility gene Os-11N3. Plant Cell 22:3864-3876.

Arlat, M., Gough, C. L., Barber, C. E., Boucher, C., and Daniels, M. J. 1991. Xanthomonas campestris contains a cluster of hrp genes related to the larger hrp cluster of Pseudomonas solanacearum. Mol. Plant-Microbe Interact. 4:593-601.

Blanvillain, S., Meyer, D., Boulanger, A., Lautier, M., Guynet, C., Denancé, N., Vasse, J., Lauber, E., and Arlat, M. 2007. Plant carbohydrate scavenging through tonB-dependent receptors: A feature shared by phytopathogenic and aquatic bacteria. PLoS One 2:e224.

Burdman, S., Shen, Y., Lee, S. W., Xue, Q., and Ronald, P. 2004. RaxH/RaxR: A two-component regulatory system in Xanthomonas oryzae pv. oryzae required for AvrXa21 activity. Mol. Plant-Microbe Interact. 17:602-612.

Büttner, D., and Bonas, U. 2002. Getting across-Bacterial type III effector proteins on their way to the plant cell. EMBO J. 21:5313-5322.

Büttner, D., and Bonas, U. 2010. Regulation and secretion of Xanthomonas virulence factors. FEMS Microbiol. Rev. 34:107-133.

Chen, L. Q. 2014. SWEET sugar transporters for phloem transport and pathogen nutrition. New Phytol. 201:1150-1155.

Chen, L. Q., Hou, B. H., Lalonde, S., Takanaga, H., Hartung, M. L., Qu, X. Q., Guo, W. J., Kim, J. G., Underwood, W., Chaudhuri, B., Chermak, D., Antony, G., White, F. F., Somerville, S. C., Mudgett, M. B., and Frommer, W. B. 2010. Sugar transporters for intercellular exchange and nutrition of pathogens. Nature 468:527-532.

Chu, Z., Fu, B., Yang, H., Xu, C., Li, Z., Sanchez, A., Park, Y. J., Bennetzen, J. L., Zhang, Q., and Wang, S. 2006. Targeting xa13, a recessive gene for bacterial blight resistance in rice. Theor. Appl. Genet. 112:455-461

Déjean, G., Blanvillain-Baufumé, S., Boulanger, A., Darrasse, A., Dugé de Bernonville, T., Girard, A. L., Carrére, S., Jamet, S., Zischek, C., Lautier, 
M., Solé, M., Büttner, D., Jacques, M. A., Lauber, E., and Arlat, M. 2013. The xylan utilization system of the plant pathogen Xanthomonas campestris pv campestris controls epiphytic life and reveals common features with oligotrophic bacteria and animal gut symbionts. New Phytol. 198:899-915.

Feng, J. X., Song, Z. Z., Duan, C. J., Zhao, S., Wu, Y. Q., Wang, C., Dow, J. M., and Tang, J. L. 2009. The xrvA gene of Xanthomonas oryzae pv. oryzae, encoding an H-NS-like protein, regulates virulence in rice. Microbiology 155:3033-3044.

Gärtner, D., Degenkolb, J., Ripperger, J. A. E., Allmansberger, R., and Hillen, W. 1992. Regulation of the Bacillus subtilis W23 xylose utilization operon: Interaction of the Xyl repressor with the $x y l$ operator and the inducer xylose. Mol. Gen. Genet. 232:415-422.

Guo, W., Zou, L. F., Li, Y. R., Cui, Y. P., Ji, Z. Y., Cai, L. L., Zou, H. S. Hutchins, W. C., Yang, C. H., and Chen, G. Y. 2012. Fructosebisphophate aldolase exhibits functional roles between carbon metabolism and the hrp system in rice pathogen Xanthomonas oryzae pv. oryzicola. PLoS One 7:e31855.

Hottes, A. K., Meewan, M., Yang, D., Arana, N., Romero, P., McAdams, H. H., and Stephens, C. 2004. Transcriptional profiling of Caulobacter crescentus during growth on complex and minimal media. J. Bacteriol. 186:1448-1461.

Huang, H. C., Hutcheson, S. W., and Collmer, A. 1991. Characterization of the hrp cluster from Pseudomonas syringae pv. syringae 61 and TnphoA tagging of genes encoding exported or membrane-spanning Hrp proteins. Mol. Plant-Microbe Interact. 4:469-476.

Ikawa, Y., Furutani, A., Ochiai, H., and Tsuge, S. 2014. StoS, a hybrid histidine kinase sensor of Xanthomonas oryzae pv. oryzae, is activated by sensing low $\mathrm{O}_{2}$ concentration and is involved in stress tolerance and virulence. Mol. Plant-Microbe Interact. 27:537-545.

Ikawa, Y., and Tsuge, S. 2016. The quantitative regulation of the hrp regulator HrpX is involved in sugar-source-dependent hrp gene expression in Xanthomonas oryzae pv. oryzae. FEMS Microbiol. Lett. 363:fnw071.

Innes, R. W., Hirose, M. A., and Kuempel, P. L. 1988. Induction of nitrogenfixing nodules on clover requires only 32 kilobase pairs of DNA from the Rhizobium trifolii symbiosis plasmid. J. Bacteriol. 170:3793-3802.

Jefferson, R. A., Kavanagh, T. A., and Bevan, M. W. 1987. GUS fusions: $\beta$-glucuronidase as a sensitive and versatile gene fusion marker in higher plants. EMBO J. 6:3901-3907.

Kametani-Ikawa, Y., Tsuge, S., Furutani, A., and Ochiai, H. 2011. An HNS-like protein involved in the negative regulation of hrp genes in Xanthomonas oryzae pv. oryzae. FEMS Microbiol. Lett. 319:58-64.

Kauffman, H. E., Reddy, A. P. K., Hsieh, S. P. Y., and Merca, S. D. 1973. An improved technique for evaluating resistance of rice varieries to Xanthomonas oryzae. Plant Dis. Rep. 57:537-541.

Lee, S. W., Jeong, K. S., Han, S. W., Lee, S. E., Phee, B. K., Hahn, T. R., and Ronald, P. 2008. The Xanthomonas oryzae pv. oryzae PhoPQ twocomponent system is required for AvrXA21 activity, hrpG expression, and virulence. J. Bacteriol. 190:2183-2197.

Li, R. F., Lu, G. T., Li, L., Su, H. Z., Feng, G. F., Chen, Y., He, Y. Q., Jiang, B. L., Tang, D. J., and Tang, J. L. 2014. Identification of a putative cognate sensor kinase for the two-component response regulator HrpG, a key regulator controlling the expression of the hrp genes in Xanthomonas campestris pv. campestris. Environ. Microbiol. 16:2053-2071.

Meisenzahl, A. C., Shapiro, L., and Jenal, U. 1997. Isolation and characterization of a xylose-dependent promoter from Caulobacter crescentus. J. Bacteriol. 179:592-600.

Niño-Liu, D. O., Ronald, P. C., and Bogdanove, A. J. 2006. Xanthomonas oryzae pathovars: Model pathogens of a model crop. Mol. Plant Pathol. 7:303-324.

O'Kane, D. J., and Prasher, D. C. 1992. Evolutionary origins of bacterial bioluminescence. Mol. Microbiol. 6:443-449.

Ochiai, H., Inoue, Y., Takeya, M., Sasaki, A., and Kaku, H. 2005. Genome sequence of Xanthomonas oryzae pv. oryzae suggests contribution of large numbers of effector genes and insertion sequences to its race diversity. Jpn. Agric. Res. Q. 39:275-287.

Ou, S. H. 1985. Bacterial Leaf Blight Rice Diseases, 2nd edition. Commomwealth Mycological Institute, Kew, U.K

Piqué, N., Miñana-Galbis, D., Merino, S., and Tomás, J. M. 2015. Virulence factors of Erwinia amylovora: A review. Int. J. Mol. Sci. 16:12836-12854.

Rajeshwari, R., Jha, G., and Sonti, R. V. 2005. Role of an in planta-expressed xylanase of Xanthomonas oryzae pv. oryzae in promoting virulence on rice. Mol. Plant-Microbe Interact. 18:830-837.

Rashid, M. M., Ikawa, Y., and Tsuge, S. 2016. GamR, the LysR-type galactose metabolism regulator, regulates $h r p$ gene expression via transcriptional activation of two key hrp regulators, HrpG and HrpX, in
Xanthomonas oryzae pv. oryzae. Appl. Environ. Microbiol. 82:39473958

Sambrook, J., Fritsch, E. F., and Maniaris, T. 1989. Molecular Cloning. A Laborarory Manual, 2nd Ed. Cold Spring Harbor Laboratory, Cold Spring Harbor, NY, U.S.A.

Schäfer, A., Tauch, A., Jäger, W., Kalinowski, J., Thierbach, G., and Pühler, A. 1994. Small mobilizable multi-purpose cloning vectors derived from the Escherichia coli plasmids pK18 and pK19: Selection of defined deletions in the chromosome of Corynebacterium glutamicum. Gene 145:69-73.

Schulte, R., and Bonas, U. 1992. A Xanthomonas pathogenicity locus is induced by sucrose and sulfur-containing amino acids. Plant Cell 4: 79-86.

Stephens, C., Christen, B., Watanabe, K., Fuchs, T., and Jenal, U. 2007. Regulation of D-xylose metabolism in Caulobacter crescentus by a LacItype repressor. J. Bacteriol. 189:8828-8834.

Swings, J., Van den Mooter, M., Vauterin, L., Hoste, B., Gillis, M., Mew, T. W., and Kersters, K. 1990. Reclassification of causal agents of bacterial blight (Xanthomonas campestris pv. oryzae) and bacterial leaf streak (Xanthomonas campestris pv. oryzicola) of rice as pathovars of Xanthomonas oryzae (ex Ishiyama 1922) sp. nov., nom. rev. Int. J. Syst Bacteriol. 40:309-311.

Takeuchi, Y., Tohbaru, M., and Sato, A. 1994. Polysaccharides in primary cell walls of rice cells in suspension culture. Phytochemistry 35:361-363.

Tsuge, S., Furutani, A., Fukunaka, R., Kubo, Y., and Horino, O. 2001. Growth complementation of hrpXo mutants of Xanthomonas oryzae pv. oryzae by virulent strains in rice cultivars resistant and susceptible to the parental strain. J. Gen. Plant Pathol. 67:51-57.

Tsuge, S., Furutani, A., Fukunaka, R., Oku, T., Tsuno, K., Ochiai, H., Inoue, Y., Kaku, H., and Kubo, Y. 2002. Expression of Xanthomonas oryzae pv. oryzae hrp genes in XOM2, a novel synthetic medium. J. Gen. Plant Pathol. 68:363-371.

Tsuge, S., Furutani, A., and Ikawa, Y. 2014. Regulatory network of hrp gene expression in Xanthomonas oryzae pv. oryzae. J. Gen. Plant Pathol 80:303-313.

Tsuge, S., Nakayama, T., Terashima, S., Ochiai, H., Furutani, A., Oku, T. Tsuno, K., Kubo, Y., and Kaku, H. 2006. Gene involved in transcriptional activation of the hrp regulatory gene hrpG in Xanthomonas oryzae pv. oryzae. J. Bacteriol. 188:4158-4162.

Tsuge, S., Ochiai, H., Inoue, Y., Oku, T., Tsuno, K., Kaku, H., and Kubo, Y. 2004. Involvement of phosphoglucose isomerase in pathogenicity of Xanthomonas oryzae pv. oryzae. Phytopathology 94:478-483.

Vidaver, A. K. 1967. Synthetic and complex media for the rapid detection of fluorescence of phytopathogenic pseudomonads: Effect of the carbon source. Appl. Microbiol. 15:1523-1524.

Warton, K., Foster, N. C., Gold, W. A., and Stanley, K. K. 2004. A novel gene family induced by acute inflammation in endothelial cells. Gene 342:85-95.

Wengelnik, K., and Bonas, U. 1996. HrpXv, an AraC-type regulator, activates expression of five of the six loci in the hrp cluster of Xanthomonas campestris pv. vesicatoria. J. Bacteriol. 178:3462-3469.

Wengelnik, K., Marie, C., Russel, M., and Bonas, U. 1996b. Expression and localization of HrpA1, a protein of Xanthomonas campestris pv. vesicatoria essential for pathogenicity and induction of the hypersensitive reaction. J. Bacteriol. 178:1061-1069.

Wengelnik, K., Rossier, O., and Bonas, U. 1999. Mutations in the regulatory gene hrpG of Xanthomonas campestris pv. vesicatoria result in constitutive expression of all hrp genes. J. Bacteriol. 181:6828-6831.

Wengelnik, K., Van den Ackerveken, G., and Bonas, U. 1996a. HrpG, a key hrp regulatory protein of Xanthomonas campestris pv. vesicatoria is homologous to two-component response regulators. Mol. Plant-Microbe Interact. 9:704-712.

Yang, B., Sugio, A., and White, F. F. 2006. Os8N3 is a host disease-susceptibility gene for bacterial blight of rice. Proc. Natl. Acad. Sci. U.S.A. 103: 10503-10508

Zhou, J., Peng, Z., Long, J., Sosso, D., Liu, B., Eom, J. S., Huang, S., Liu, S., Vera Cruz, C., Frommer, W. B., White, F. F., and Yang, B. 2015. Gene targeting by the TAL effector PthXo 2 reveals cryptic resistance gene for bacterial blight of rice. Plant J. 82:632-643.

\section{AUTHOR-RECOMMENDED INTERNET RESOURCES}

National Center for Biotechnology Information web server: http://blast.ncbi.nlm.nih.gov/Blast.cgi

The Osaka University Gen-Info web server:

http://www.gen-info.osaka-u.ac.jp/testdocs/tomocom//t-ken-e.html 